\title{
Are probiotics useful in the treatment of chronic idiopathic constipation in adults? A review of existing systematic reviews, meta-analyses, and recommendations
}

\author{
Mikołaj Kamiński ${ }^{1}$, Karolina Skonieczna-Żydecka², Igor Łoniewski ${ }^{2}$, Anastasios Koulaouzidis ${ }^{3}$, Wojciech Marlicz \\ ${ }^{1}$ Sanprobi Sp. z o.o. sp. k., Szczecin, Poland \\ ${ }^{2}$ Department of Human Nutrition and Metabolomics, Pomeranian Medical University, Szczecin, Poland \\ ${ }^{3}$ Centre for Liver and Digestive Disorders, Royal Infirmary of Edinburgh, Scotland, UK \\ ${ }^{4}$ Department of Gastroenterology, Pomeranian Medical University, Szczecin, Poland
}

Gastroenterology Rev 2020; 15 (2): 103-118

DOI: https://doi.org/10.5114/pg.2019.86747

Key words: chronic idiopathic constipation, irritable bowel syndrome, meta-analysis, microbiota, probiotics, recommendations, systematic review.

Address for correspondence: Wojciech Marlicz, Department of Gastroenterology, Pomeranian Medical University, 1 Unii Lubelskiej St, 71-252 Szczecin, Poland, phone: +48 608296 989, e-mail: marlicz@hotmail.com

\begin{abstract}
Chronic idiopathic constipation (CIC) has emerged as common problem for contemporary gastroenterology and is one of the most frequent complaints in primary care. Chronic idiopathic constipation significantly affects patients' quality of life and has an impact on global health and economy. Functional gastrointestinal disorders and bowel disorders, according to Rome IV criteria, result from inappropriate gut-brain interactions. The pathophysiology is complex and poorly understood, with evidence accumulating that gut microbiota can be implicated in the development and function of the enteric nervous system. Gut bacteria modulate gut barrier function, short chain fatty acid synthesis, and bile acid metabolism, factors which play roles in the gut peristalsis regulation. The high prevalence of CIC, with poor treatment outcomes, warrants searches for new forms of therapy, including probiotic therapies. Probiotics are often recommended by medical practitioners, but evidence-based utility in adults with CIC is uncertain. Recommendations/guidelines are often based on results from individual studies, rather than meta-analyses or umbrella reviews. Additionally, meta-analyses often indicate a group of probiotics rather than individual strains, and they create difficulty for physicians in making therapeutic choices. More CIC patient randomised clinical studies utilising well-defined strains, or combinations, are necessary.
\end{abstract}

\section{Introduction}

Chronic idiopathic constipation (CIC) is one of the most frequent complaints in primary care [1]. After excluding secondary causes of constipation, arising from mechanical obstacles, neurodegenerative and neurologic disorders, neuroendocrine diseases, electrolyte disturbances, and drug-related adverse events [2], CIC disorders can be classified as: i) functional defecation disorder (FDD), further sub-classified as inadequate defecatory propulsion or dyssynergic defecation; ii) slow-transit constipation (STC), and iii) normal transit constipation, further subclassified as functional constipation (FC) and constipation-predominant irritable bowel syndrome (IBS-C). These classifications are not mutually exclusive, and significant overlap exists [3]. It has been estimated that CIC was the leading problem identified in a patient visit from more than three billion patient visits yearly in medical centres in the United States $[4,5]$. The annual cost of treatment of CIC ranges from $\$ 1912$ to $\$ 7522$ per patient, while treatment of a patient with IBS-C costs around \$1356 [6]. Almost half of patients with symptoms of $\mathrm{CIC}$ are not satisfied after medical advice and therapy, mostly due to lack of therapeutic efficacy and uncertainty concerning its safety [7].

The pathophysiology of $\mathrm{CIC}$ is complex and not well understood. The following mechanisms have been implicated in its pathogenesis: i) gastrointestinal motor 
dysfunction, ii) slow colonic transit in STC, iii) inadequate peristaltic movements, iv) failure in smooth muscle relaxation, $v$ ) overactivity of the colonic wall, and vi) microbiota and gut-brain axis (GBA) alterations [2].

Patients with normal colonic transit constipation represent the most prevalent subgroup of $\mathrm{CIC}$ with unclear pathophysiology. Patients with FDD represent the second most common group of CIC disorders, with paradoxical anal contraction, failure or impairment of anal relaxation, or inadequate rectal and abdominal propulsive forces implicated in pathogenesis [8]. Patients with STC are the least prevalent CIC subgroup with limited or absent increase in postprandial motor activity and impaired retrograde colonic propulsion [8].

The high prevalence of $\mathrm{CIC}$ and low or moderate treatment efficacies warrant the development of new forms of therapy. Among various therapeutic methods in patients with functional gastrointestinal disorders (FGIDs), probiotics are gaining popularity and have become widely used in clinical practice $[9,10]$. To show a clinical relationship between a studied probiotic at a certain dose, clinicians have to evaluate its effect size and duration of action. Determination of replicability and reproducibility of each finding, the biological likelihood, and potential explanation of proposed interactions and alternatives are of particular interest [11] Finally, it is necessary to evaluate how the discovered relationship conforms with current knowledge.

Because differences in interpretations of epidemiological findings can exist between various experts and authorities, well-powered, appropriately-designed studies, preferably with a high level of evidence (i.e. systematic reviews and meta-analyses), are essential to draw conclusions regarding causation and to develop guidelines and recommendations.

Unfortunately, it should be noted that high-quality data from nutrition-related interventions rarely exist. In parallel, as stated by the expert panel of the International Scientific Association for Probiotics and Prebiotics (ISAPP), bacteria-containing food and supplements should be evaluated as food and dietary supplements overall [12]. Because no probiotic claims for probiotics in foods in the European Union (EU) have been judged to be sufficiently substantiated, medical authorities now recommend microbial supplements on the basis of scientific literature and recommendations published by health-related practitioners.

In the last decade only a few meta-analyses and recommendations evaluating utility of probiotics in $\mathrm{CIC}$ have been published. Several conclusions conflicted, making judgment difficult. There was also great uncertainty among medical professionals as to the choice of an adequate interventional protocol. The aim of the present review is to update readers concerning possible mechanisms of the action of probiotics in CIC and to analyse published systematic reviews, meta-analyses, and recommendations regarding their effectiveness in adults with $\mathrm{CIC}$.

\section{CIC diagnosis: The Rome IV criteria for functional constipation, constipation-predominant irritable bowel syndrome, and functional defecation disorders}

FGIDs and bowel disorders (BDs), according to the Rome IV diagnostic criteria, result from improper gutbrain interactions. FGIDs and BDs are currently defined as a group of disorders classified by gastrointestinal symptoms related to any combination of: i) motility disturbances, ii) visceral hypersensitivity, iii) altered mucosal and immune function, iv) gut microbiome, and/or v) central nervous system processing [13]. The Rome IV criteria introduced a modern definition of functional manifestation of the disease on the basis of its pathophysiology rather that its non-organic cause [14].

Functional constipation (FC) is a functional bowel disorder of difficult, infrequent, or incomplete defecation [15]. In 2007, using the Rome II criteria, Choung et al. identified that a 12-year cumulative incidence of constipation was as high as 17\% [16]. Female gender, reduced caloric intake, and age over 50 years were recognised as pivotal risk factors of this condition [17, 18]. However, the terminology and definitions of FC are not always appropriate. In this regard it is noteworthy that Rome IV criteria do not use the term "chronic idiopathic constipation". However, this term does appear in many studies [19] and can be viewed as an umbrella term for all functional defecation disorders. Brandt et al. defined $\mathrm{CIC}$ as the presence of unsatisfactory defecation for at least 3 months and characterised by infrequent stools, difficult stool passage, or both [20]. This definition does not correspond to a category in the FC Rome IV criteria, but patients diagnosed with $\mathrm{CIC}$ are frequently considered as patients with FC [21]. Because the use of multiple definitions of $\mathrm{CIC}$ may lead to conceptual confusion, researchers are strongly encouraged to use Rome IV criteria for definitions and terminology for clinical trials and clinical assessment of participants suffering from chronic constipation.

Constipation-predominant irritable bowel syndrome (IBS-C) is a subtype of IBS in which pain, distension, bloating, and constipation [15] are predominant symptoms of the disease (Table I). The global prevalence of IBS was estimated to be around 11\%, with almost 30\% as cases of IBS-C. The incidence was higher in women and individuals below 50 years of age [22-24]. Clinical 
Table I. Diagnostic criteria for functional constipation (FC) and constipation-predominant irritable bowel syndrome (IBS-C) [15]

\begin{tabular}{|c|c|c|}
\hline Condition & FC & IBS-C \\
\hline Risk factors & $\begin{array}{l}\text { Female gender, reduced caloric intake, } \\
\text { age }>50 \text { years }\end{array}$ & Female gender, age $<50$ years \\
\hline Rome IV criteria & $\begin{array}{l}\text { 1. Two or more of: } \\
\text { a. Straining during }>1 / 4 \text { ( } 25 \%) \text { of defecations } \\
\text { b. Lumpy or hard stools in }>1 / 4 \text { ( } 25 \%) \text { of } \\
\text { defecations } \\
\text { c. Sensation of incomplete evacuation in }>1 / 4 \\
\text { ( } 25 \%) \text { of defecations } \\
\text { d. Sensation of anorectal obstruction/blockage in } \\
>1 / 4 \text { ( } 25 \% \text { ) of defecations } \\
\text { e. Manual manoeuvres to facilitate }>1 / 4 \text { ( } 25 \%) \text { of } \\
\text { defecations (e.g., digital evacuation, support of } \\
\text { the pelvic floor) } \\
\text { f. Fewer than } 3 \text { spontaneous bowel movements } \\
\text { per week } \\
\text { 2. Loose stools are rarely present without the use } \\
\text { of laxatives } \\
\text { 3. Insufficient criteria for irritable bowel syndrome }\end{array}$ & $\begin{array}{l}\text { IBS: } \\
\text { Recurrent abdominal pain, on average, at least } \\
1 \text { day per week in the last } 3 \text { months, associated } \\
\text { with } 2 \text { or more of the following criteria: } \\
\text { 1. Related to defecation. Associated with a change } \\
\text { in the frequency of stool } \\
2 \text {. Associated with a change in form of stool } \\
\text { IBS-C: } \\
\text { a. }>1 / 4(25 \%) \text { of bowel movements with Bristol } \\
\text { stool form type } 1 \text { or } 2 \text { [R } 8 \text { ] } \\
\text { b. }<1 / 4(25 \%) \text { of bowel movements with Bristol } \\
\text { stool form types } 6 \text { or } 7 \\
\text { Alternative } \\
\text { Patients reports that abnormal bowel movements } \\
\text { are usually constipation }\end{array}$ \\
\hline Symptoms duration & $\begin{array}{l}\text { Criteria fulfilled for the last } 3 \text { months with } \\
\text { symptom onset at least } 6 \text { months prior to } \\
\text { diagnosis }\end{array}$ & $\begin{array}{l}\text { Criteria fulfilled for the last } 3 \text { months with } \\
\text { symptom onset at least } 6 \text { months prior to } \\
\text { diagnosis }\end{array}$ \\
\hline
\end{tabular}

differentiation between FC and IBS-C may introduce many difficulties as the diagnoses overlap [25-28]. Thus, FC and IBS-C should be considered as part of a continuous spectrum of disorders rather than isolated diseases [15, 29] (Figure 1, Table I). Patients with FDD may fulfil the Rome IV symptom criteria for either FC or IBS-C. The criteria for FDD also require the presence of at least two out of three independent clinically-validated physiological tests: i) abnormal balloon expulsion, ii) an imaging study documenting improper evacuation, and iii) anal manometry or surface electromyographic activity (EMG) documenting abnormal anorectal evacuation [30].

\section{CIC pathophysiology}

Pathophysiology of constipation in functional bowel disorders has a multifactorial origin. As a family history of chronic constipation has been reported, $\mathrm{CIC}$ is thought to have a genetic background [31, 32], but data concerning this are scarce. Genes suggested to be involved in constipation include a membrane-bound bile acid receptor, TGR5 (also known as GPBAR1) [33], as well as the $\alpha$-subunit of the voltage-gated sodium channel NaV1.5, namely SCN5A [34]. Limited data show that the average rate of penetration of genetic changes in the global population is difficult to assess.

It has been confirmed that in STC individuals abnormal motility may result from skewed serotonin signalling [25], a decreased level of $P$ substance in enteric nervous system in mucosa and submucosa [35], low neural density in myenteric plexus, excessive count of nitric oxide-positive neurons and low count of vasoactive intestinal peptide-positive neurons [36], changes within colonic endocrine cell composition [37], and/or diminished volume of colon interstitial cells [38, 39]. These may all result in altered gastrointestinal (GI) motility, visceral hyperalgesia, immune activation, and increased intestinal permeability. Altered intestinal microbiome composition allows improper communication within the GBA and thus may be involved in the aetiol-

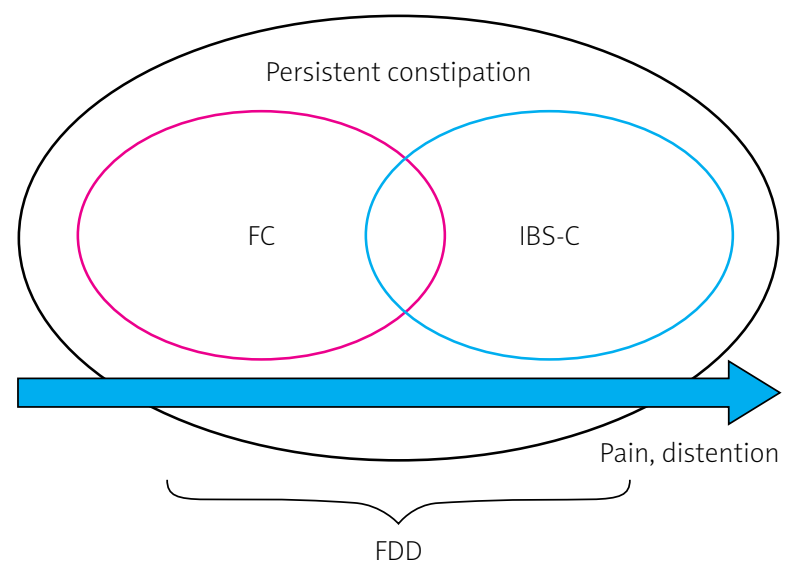

Figure 1. Simplified overview of chronic idiopathic constipation (CIC) and overlapping criteria for functional constipation (FC), irritable bowel syndrome with predominant constipation (IBS-C), and functional defecation disorder (FDD) 
ogy of the condition [14]. In fact, diverse gut microbiota are essential for many physiological processes, including immune response and GI function [40]. Neuroactive molecules produced within the gut ecosystem, via auto, para-, or endocrine mechanisms, influence mucosal secretion, smooth muscle motility, and intestinal blood flow. Transmission of neural signals via vagal afferent nerves and interneurons close the gut-brain communication circle [41].

\section{Gut microbiome alteration in constipation}

The human intestine forms a habitat for more than 1000 different species of microorganisms, predominantly bacteria, hence the number of microbiotic cells is nearly equal to the number of host's cells $[42,43]$. There is an increasing body of evidence that alteration of gut microbiota may contribute to the development of functional bowel disorders, which may be secondary to gut microbiota dysbiosis responsible for altered metabolic activity [15]. The putative microbiotic-dependent mechanisms in chronic constipation are presented in Figure 2.

In experimental and clinical studies, changes in microbiota associated with the occurrence of FC have been observed [44]. A direct relationship between microbiota and constipation was demonstrated in an experiment conducted by Ge et al. [19]. In this rodent study a 4-week, broad-spectrum, antibiotic therapy was followed by faecal microbiome transplantation (FMT) from constipated or healthy donors [19]. Mice receiving transplants from constipated donors were more likely to develop constipation in comparison to the control group. The authors evaluated microbiotic metabolites and found that short-chain fatty acids (SCFAs) and secondary bile acids (sBAs) were decreased in mice transplanted [45] with faeces collected from constipated humans.

However, the results of experimental investigations have not been confirmed by direct observations in human studies. Recently, an elegant paper authored by Ohkusa et al. summarised gut microbiotic fingerprints in constipated patients [44]. The report covered patients diagnosed with both FC and IBS-C. Different methods of microbiotic analyses were utilised in recruited patient cohorts. Historically these were culture dependent, whereas more recently sequence-based genetic and fluorescence in situ hybridisation (FISH) techniques have been used, together making it extremely difficult to pool results and draw conclusions. For instance, Khalif et al. found that patients diagnosed with FC had lower abundance of Bifidobacterium, Lactobacillus, Clostridium, and Bacteroides and elevated counts of Enterobacteriaceae (namely E. coli) and S. aureus along with fungi

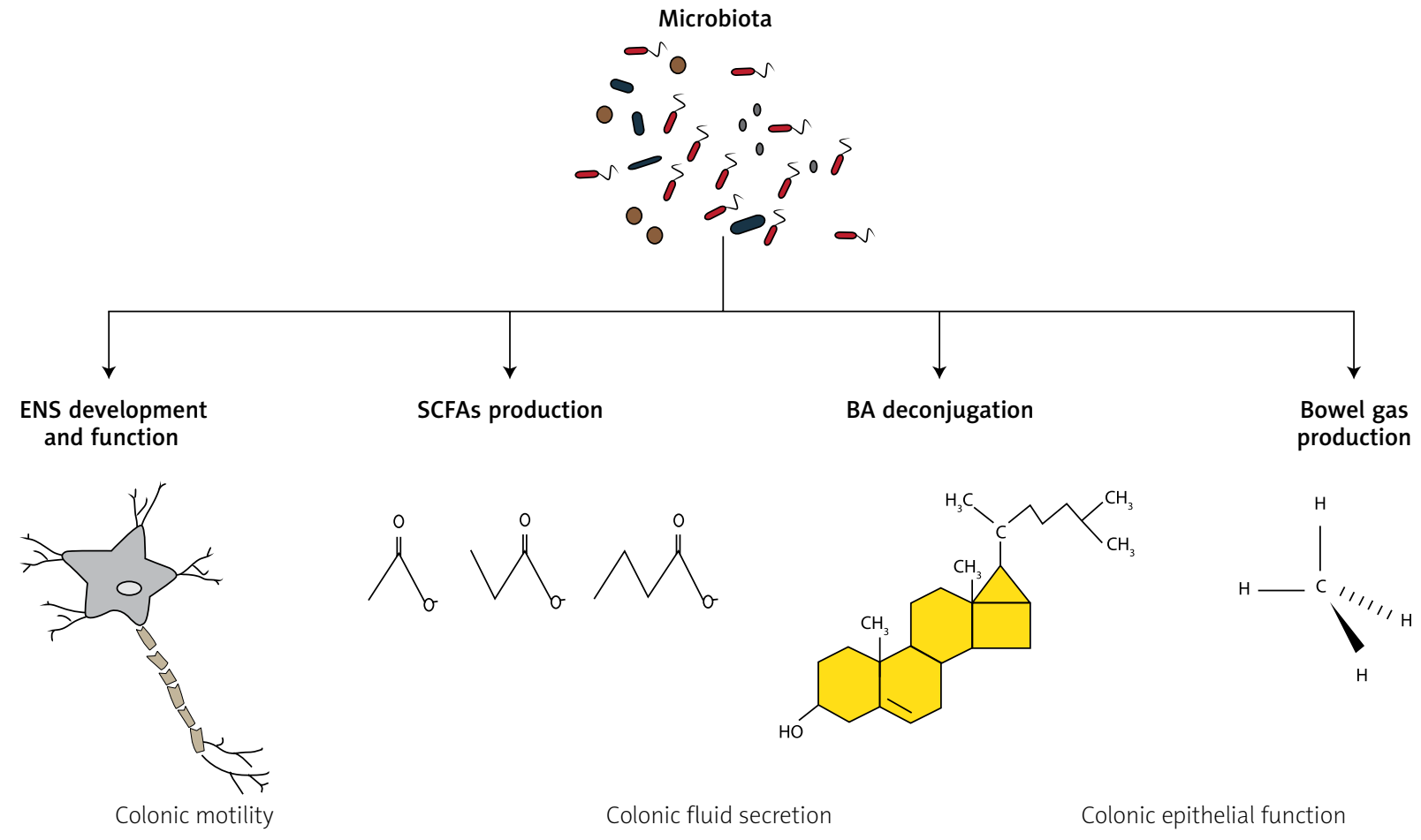

Constipation

Figure 2. Putative microbiotic-dependent mechanisms in chronic constipation $B A$ - bile acids, ENS - enteric nervous system, SCFAs - short-chain fatty acids. 
[46]. However, these results were based on microbial culture analysis of faecal samples. Similarly, Kim et al. found that patients diagnosed with FC had significantly diminished counts of Bifidobacterium and Bacteroides compared to healthy controls [47]. In patients suffering from IBS-C the most prevalent cases had lower faecal abundance of Actinobacteria, including Bifidobacteria, along with higher counts of Bacteroidetes in gut mucosa. All in all, there are no consistent findings concerning typical gut microbiotic alterations for constipated patients. Currently, faecal microbiotic alterations cannot be used as a marker for constipation or as a treatment marker. More studies, characterising not only skewed bacterial abundance but also with dysbiotic metrics such as $\alpha$ - and $\beta$-diversity and consequent descriptions of disrupted metabolic functioning of the microbiome, are necessary, especially in constipated patients stratified according to clinical indices such as effectiveness of treatment [48]. New hope should also be directed towards new methods of microbiome analysis including measurement of its function, i.e. whole genome sequencing and use of the Kyoto Encyclopedia of Genes and Gene Systems (KEGG), ortholog prediction [49] using the Phylogenetic Investigation of Communities by Reconstruction of Unobserved States (PICRUSt) [50], as well as the assessment of metabolic activity of microbiota (e.g. production of short-chain fatty acids) [51].

\section{Possible involvement of microbiota in chronic constipation}

Gut microbiota affect the structure and function of the central nervous system due to interactions with enterochromaffin cells and vagal afferent nerve pathways $[52,53]$. Rome IV criteria emphasise that the gut-brain axis may be involved in the aetiology of functional bowel disorders [15]. These pathways might serve as potential targets for future interventions.

Intestinal bacteria affect gut motility and are involved in enteric nervous system (ENS) development, SCFA synthesis, and bile acid metabolism $[54,55]$. Bacterial colonisation of germ-free mice provide key factors for understanding the development of the ENS [56]. Agitation within the ENS is transmitted via fast-acting catecholamines and slow-acting neuropeptides. Additionally, the inflammatory response - as a consequence of disruption within the gut microbiome and thus the intestinal barrier - is influenced by sensory neurons. This neural activity may originate from neurogenic inflammation (by means of vasodilatation and plasma extravasation) and independently increase the synthesis of neuropeptides $[57,58]$. Norepinephrine increases the pathogenic properties of bacteria and viruses rendering them susceptible to dendritic cells, which

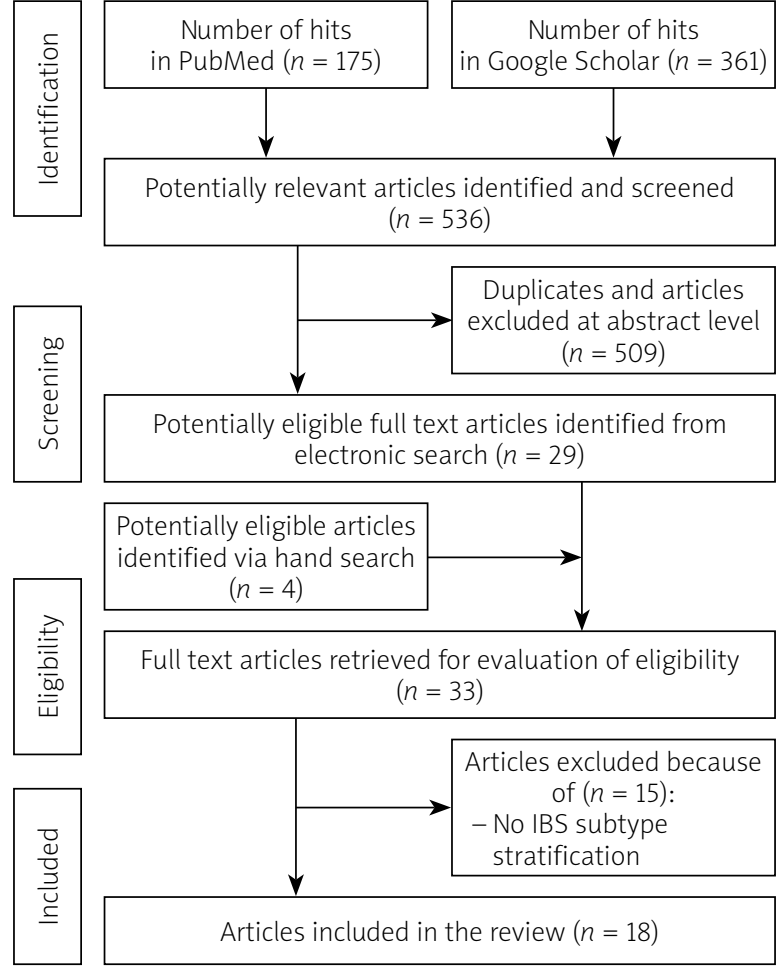

Figure 3. Flow chart

consequently increases the intensity of inflammation [59]. To close the circle, different gut microbiotic metabolites regulate the function of the myenteric plexus, thus affecting visceral perception, motility, as well as secretory and motor functions of the Gl tract [60-63]. For example, SCFAs stimulate colonic blood flow and gut motility [64]. Products of metabolism from colonic anaerobic bacteria, such as acetate, propionate, and butyrate, stimulate ileal propulsive contractions as a result of serotonin secretion [65]. Furthermore, bacterial bile acid metabolites, i.e. deconjugated bile salts, may stimulate colonic motor response [66]. SCFA and BA levels are altered in patients with FC and/or IBS-C. Currently, there is evidence that the SCFA level is typically increased [67], and BA decreased [68], among constipated patients. Lastly, environmental stimuli, including psychological stress, have been recognised as gut-barrier integrity disruptors [69].

Another possible link between constipation and microbiota may be small intestinal bacterial overgrowth (SIBO) [70]. SIBO was shown to be associated with prolonged small bowel transit time [71]. Sarosiek et al. observed that in patients with chronic constipation, lubiprostone increased the frequency bowel movements. Moreover, $41 \%$ of patients who were recognised as SIBO-positive became SIBO-negative after treatment [72]. In the aforementioned study, all SIBO-positive patients were tested positive for both methane and hy- 


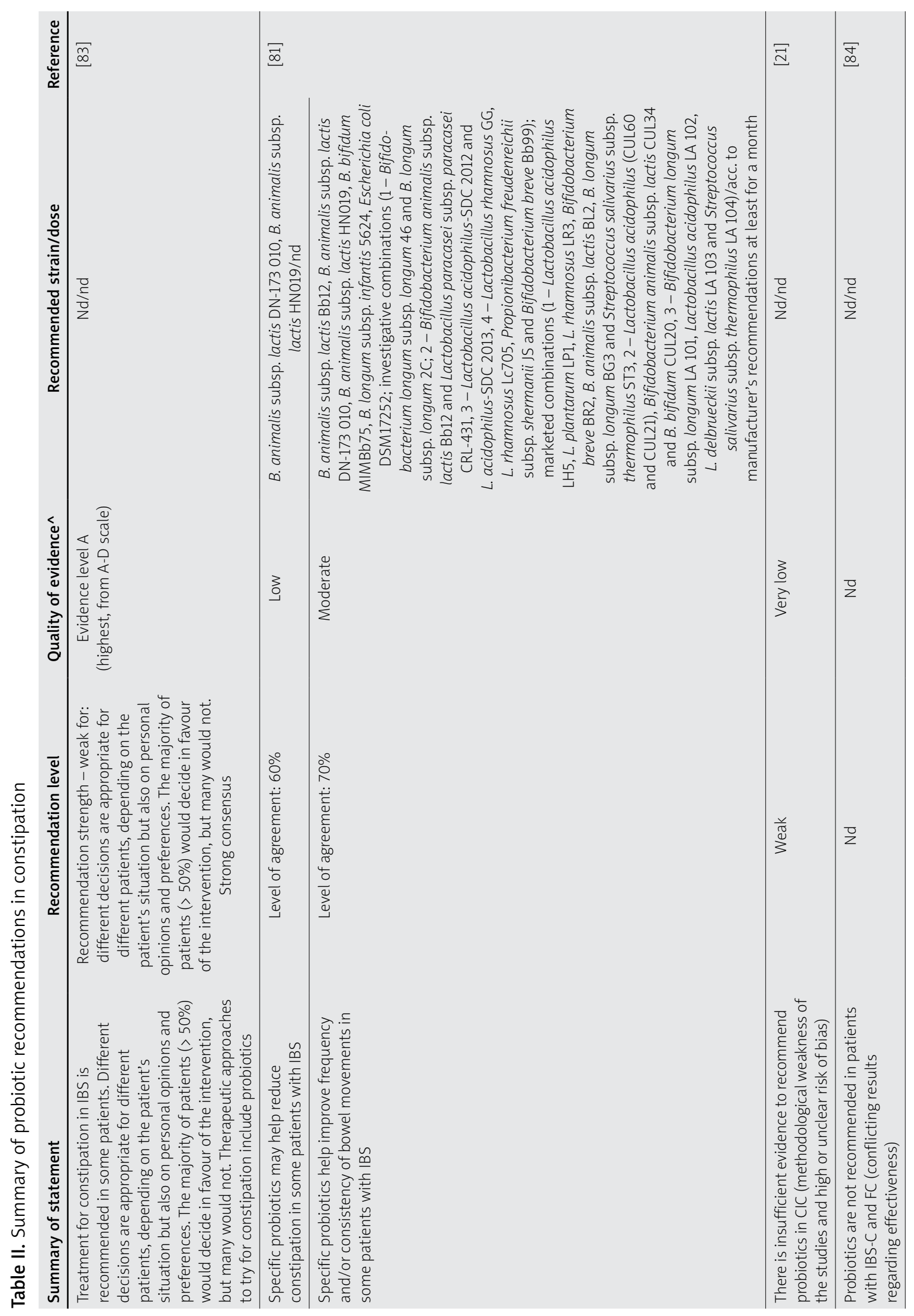




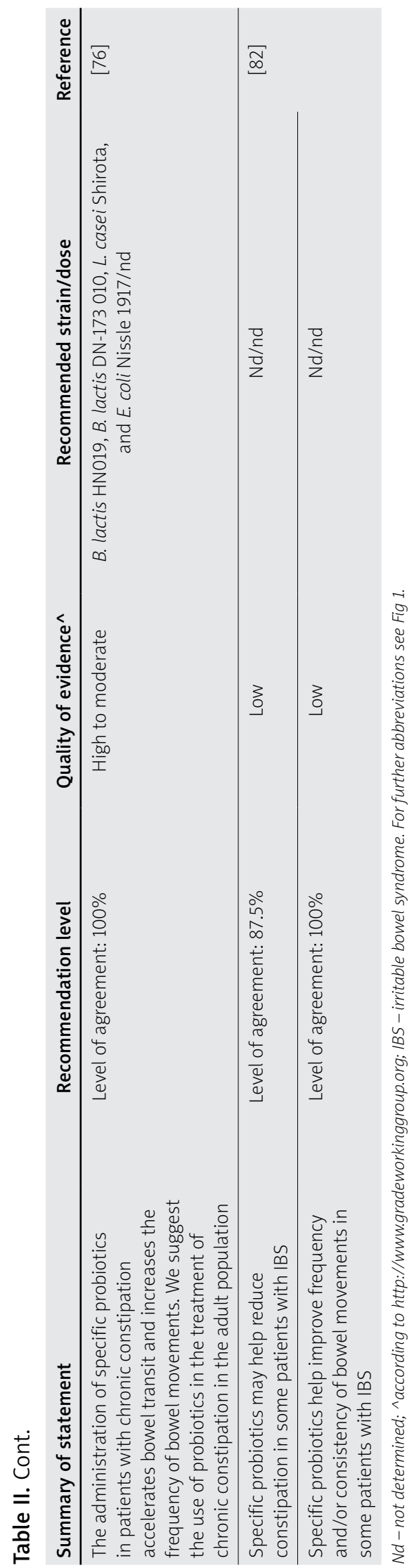

drogen in breath tests. Therefore, both methane and hydrogen may contribute to constipation in SIBO-positive individuals [72]. However, Grover et al. reported that methane alone, regardless of the presence of SIBO, was linked to IBS-C [73]. It is likely that SIBO enhances constipation via methane and hydrogen production. Of note, SIBO might arise secondarily to diminished intestinal clearance in patients with decreased bowel motility. All of the above may accelerate a circle of constipation-related causes, although these interactions require further investigation.

\section{Probiotics in CIC treatment}

Because FGIDs are associated with improper signalling within the GBA, the microbiome may provide a guide towards therapies to counteract or relieve constipation. Probiotics contain live microorganisms, which when administered in adequate amounts confer a health benefit to the host [74]. Probiotics have been used successfully in patients with various FGIDs, and some recommendations concerning use of probiotics in clinical practice are already available $[9,75]$. However, their use in constipated individuals is still controversial. The effects of probiotics are modest and depend on the strain and the available meta-analyses cover data from interventions with both single-strain or multi-strain probiotic formulations.

In addition, probiotic dose and timing of administration vary among reported clinical trials. In particular, the number of bacteria colony-forming units (CFU) in the probiotic formulations were neither assessed nor confirmed in most clinical interventions conducted. Medical practitioners often recommend probiotics by rebound to patients demands and/or on the basis of available internet recommendations.

To assess the efficacy of probiotics in constipation, we analysed the results of systematic reviews and metaanalyses in this field. We also compared the results of meta-analyses with available guidelines and recommendations published so far.

\section{Systematic review of literature}

We conducted PubMed and Google Scholar searches using the following search strings: 1 . (constipation OR IBS OR IBS-C OR functional) AND probiotics AND (recommendations OR guideline OR meta-analysis OR systematic review) and 2. title: probiotic AND guidelines, to evaluate the opinion of health-related authorities toward probiotics in constipated patients. The electronic search was supplemented by a manual review of the reference lists from eligible publications and relevant reviews. The search was carried out from the databases' creation until 15.04.2019. 
Our inclusion criteria were as follows:

1) documents (recommendations/guidelines/metaanalyses/systematic reviews), in which the effectiveness of probiotics in patients with constipation/ FGIDs/healthy persons with infrequent bowel movements was analysed;

2) documents (recommendations/guidelines/metaanalyses/systematic reviews) in humans;

3) documents in English/Polish. Exclusion criteria were:

1) reports with no constipation-related outcome;

2) reports in patients with diarrhoea-predominant IBS;

3) reports in which patients with IBS were not stratified into subtypes and documented no constipation-related outcomes.

\section{Results of systematic search}

The initial number of publications found (hits) were 536. After the title and abstract review and removal of duplicates, we included 33 papers for the full-text analysis phase. Finally, we extracted data from 18 publications (for details see flowchart in Figure 3 ). The results from guidelines and recommendations $(n=10)$ on probiotic utility in constipation are presented in Tables
II and III. In parallel we compared the results of metaanalyses/systematic reviews $(n=8)$ that evaluated the efficacy of probiotics in constipation.

We obtained data on the number of participants, duration of probiotic intervention, doses of probiotics, and the names of the strains that were used. Additionally, we noted the main results and conclusions and most importantly the trial quality indices (risks of bias). Only data on constipation-related study characteristics and outcomes were abstracted. In case of more than two study arms, data were abstracted separately for probiotic doses. The details are given in Table IV. Among 10 papers comprising guidelines and recommendations, six evaluated probiotic efficacy in patients diagnosed with chronic/functional constipation [21, 76-80] and four concerned constipation-related outcomes in patients with IBS-C $[15,81-83]$.

There were two recommendation papers that did not find any reasons to use probiotics as a treatment option [21, 84], four documents in which authors concluded that probiotics may be beneficial but in only a subgroup of patients [76, 81-83], and four which provided exact probiotic strains as effective in treatment of constipation using levels of evidence based on the

Table III. Summary of probiotic guidelines in constipation

\begin{tabular}{|c|c|c|c|}
\hline Strain & Dose & $\begin{array}{l}\text { Level of } \\
\text { evidence }^{*}\end{array}$ & Reference \\
\hline $\begin{array}{l}\text { Bifidobacterium bifidum (KCTC 12199BP), B. lactis (KCTC 11904BP), } \\
\text { B. longum (KCTC 12200BP), Lactobacillus acidophilus (KCTC } \\
11906 \mathrm{BP}) \text {, L. rhamnosus (KCTC 12202BP), and Streptococcus } \\
\text { thermophilus (KCTC 11870BP) }\end{array}$ & $2.5 \times 10^{8} \mathrm{CFU} /$ day & $\| I^{*}$ & {$[80]$} \\
\hline Lactobacillus reuteri DSM 17938 & $1 \times 10^{8} \mathrm{CFU} /$ twice daily & $\| I^{*}$ & \\
\hline L. reuteri DSM 17938 & 10/tab; 1 tab/day & $1^{*}$ & {$[77]$} \\
\hline $\begin{array}{l}\text { Combination of the following strains: L. acidophilus SD5212, L. casei } \\
\text { SD5218, L. bulgaricus SD5210, L. plantarum SD5209, B. longum } \\
\text { SD5219, B. infantis SD5220, B. breve SD5206, S. thermophilus } \\
\text { SD5207 }\end{array}$ & $45 \times 10^{10} /$ sachet; $1-4$ sachets/day & \|\|$^{\star}$ & \\
\hline L. reuteri DSM 17938 & 10/tab; 1 tab/day & 1 & {$[78]$} \\
\hline B. (animalis) lactis CNCMI-2494 & 10/lq; 1-3 servings/day & I & \\
\hline $\begin{array}{l}\text { Combination of the following strains: L. acidophilus SD5212, L. casei } \\
\text { SD5218, L. bulgaricus SD5210, L. plantarum SD5209, B. longum } \\
\text { SD5219, B. infantis SD5220, B. breve SD5206, S. thermophilus } \\
\text { SD5207 }\end{array}$ & $45 \times 10^{10} /$ sachet; $1-4$ sachets/day & ॥ & \\
\hline L. reuteri DSM 17938 & 10/tab; 1 tab/day & I & {$[79]$} \\
\hline B. (animalis) lactis CNCMI-2494 & 10/lq; 1-3 servings/day & 1 & \\
\hline $\begin{array}{l}\text { L. acidophilus DSM24735, L. paracasei DSM24733, L. delbrueckii } \\
\text { subsp. bulgaricus DSM24734, L. plantarum DSM24730, } \\
\text { B. longum DSM24736, B. infantis DSM24737, B. breve DSM24732, } \\
\text { S. thermophilus DSM24731 }\end{array}$ & $\begin{array}{l}45 \times 10^{10} / \text { sachet; } 1-2 \text { sachets/day or } \\
90 \times 10^{10} / \text { sachet; } 1 \text { sachet/day }\end{array}$ & ॥ & \\
\hline
\end{tabular}




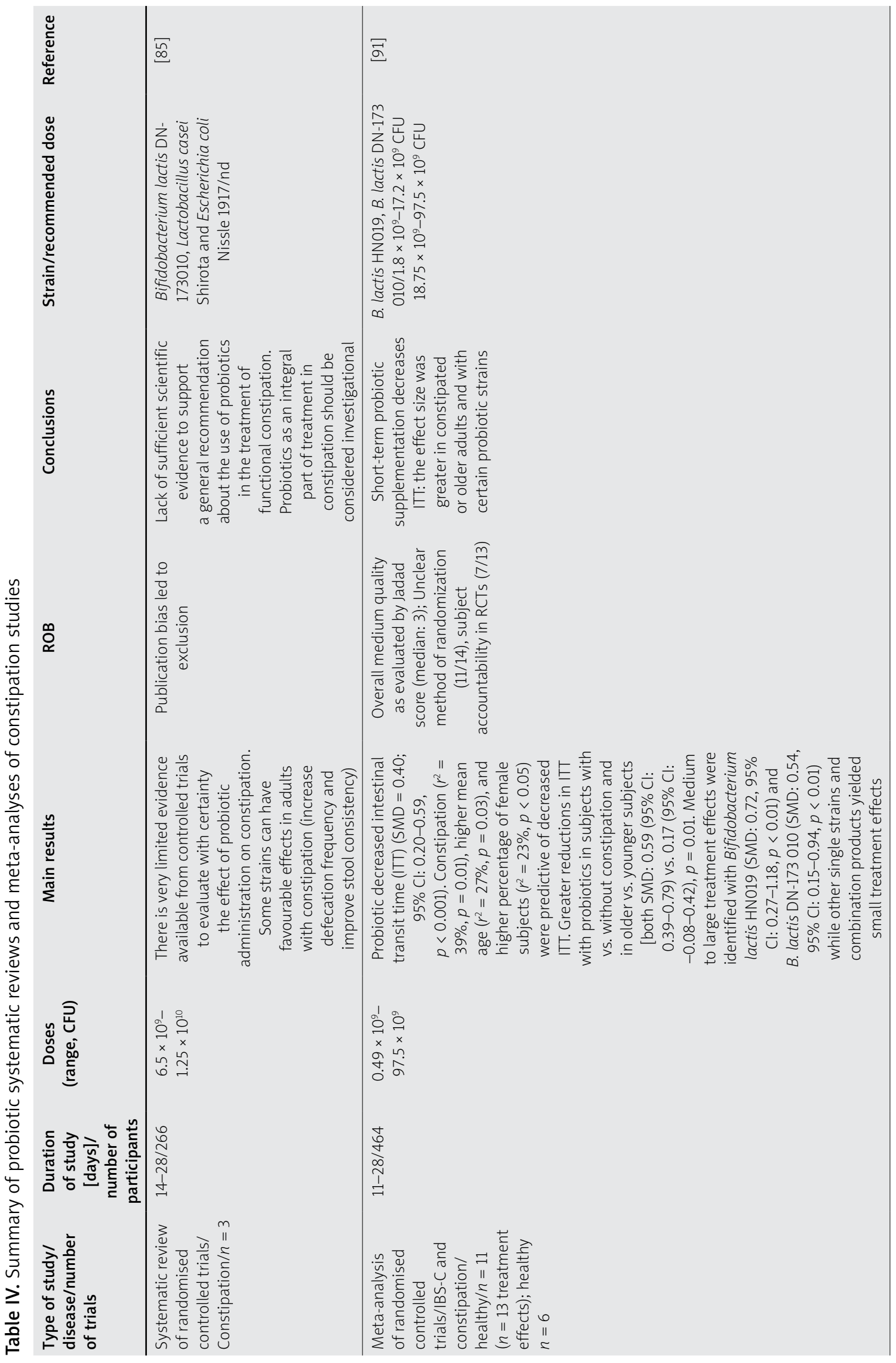




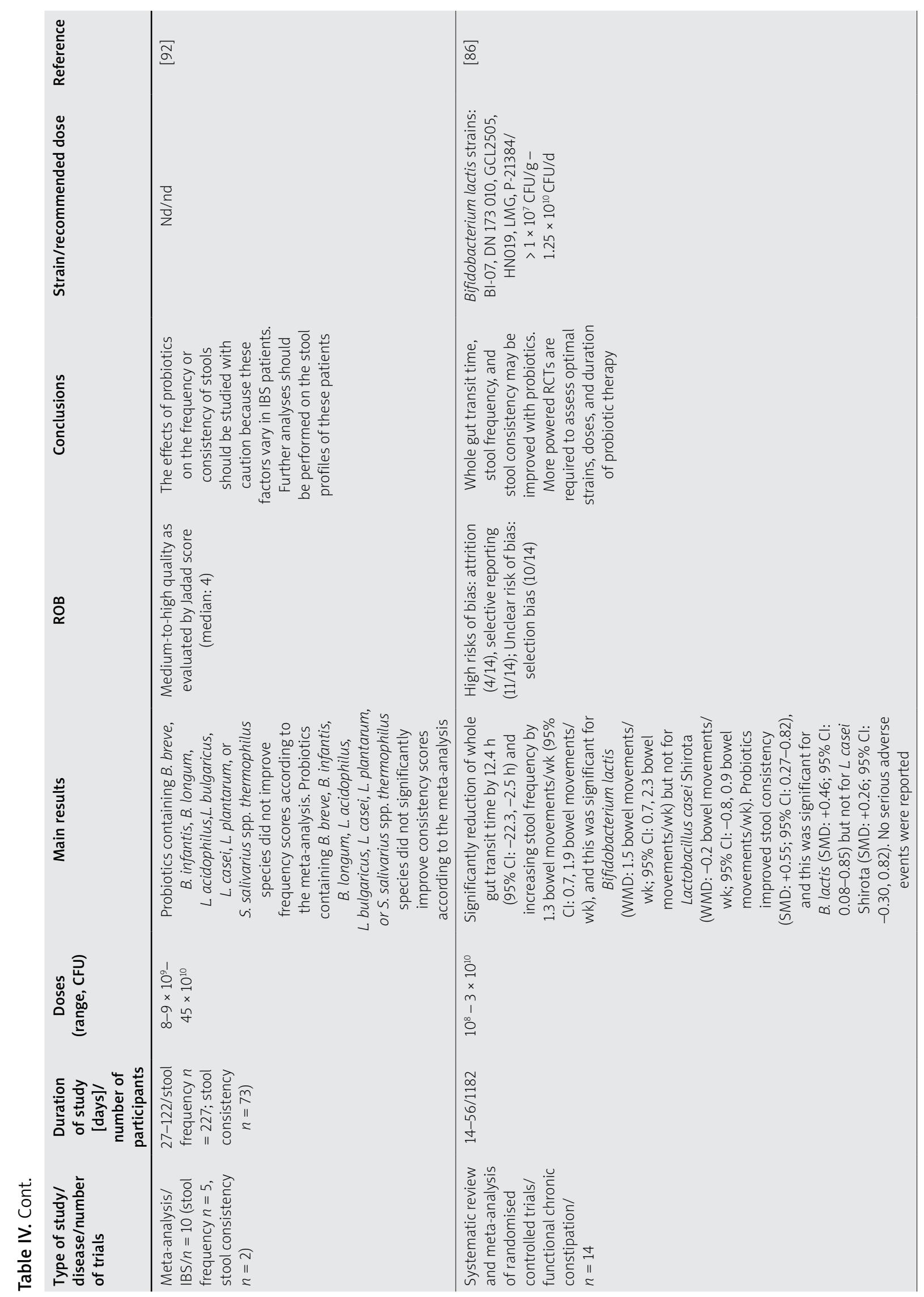




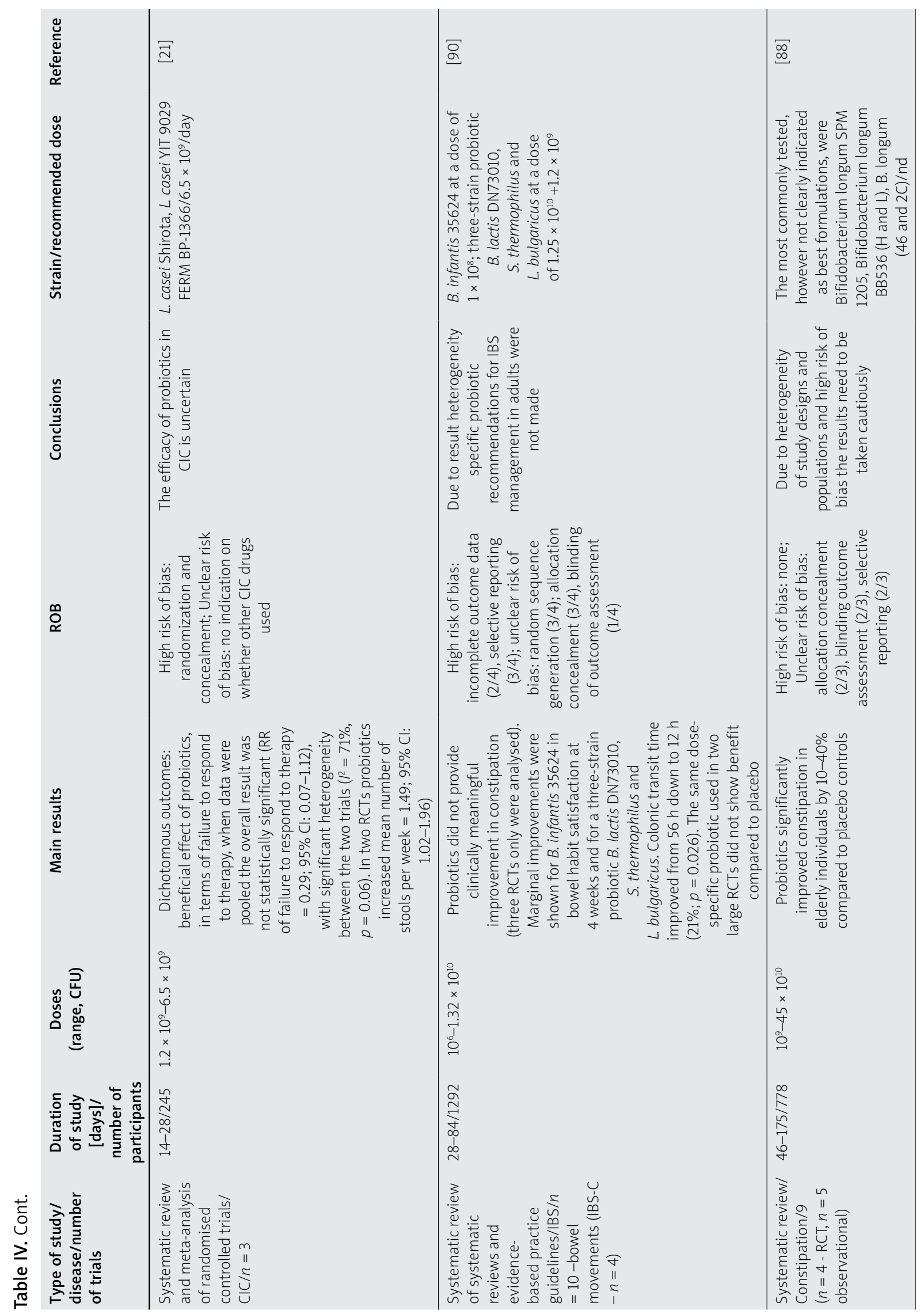




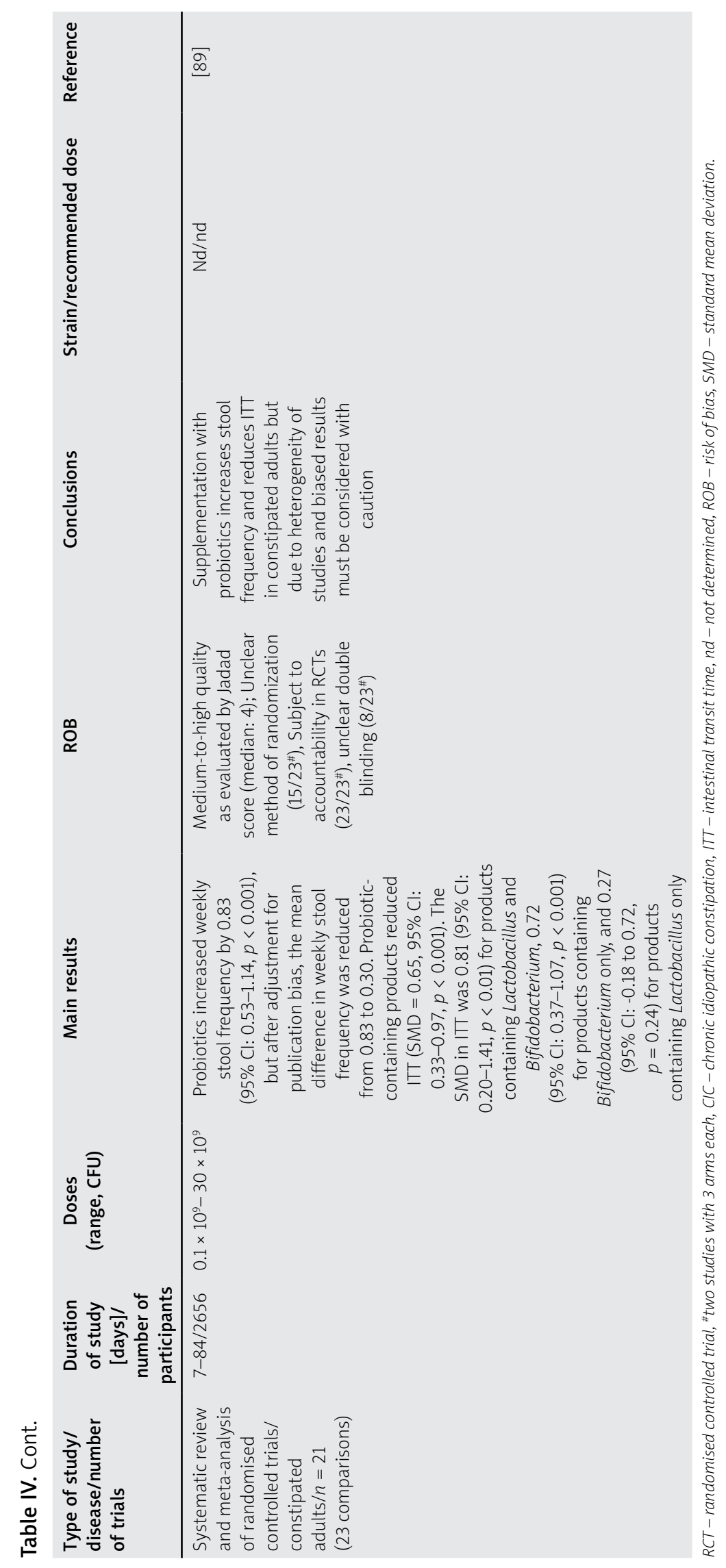


design of clinical trials (level I - randomised clinical trials (RCT), level III- nonrandomised studies). Among meta-analyses and systematic reviews there were five papers with studies conducted in a population of patients with constipation [85-89] and three in persons diagnosed with IBS-C [90-92]. Out of six studies evaluating probiotic therapy as an integral part of constipation treatment, the results of four were negative [ 85 , $87,90,92]$ and two were positive $[88,91]$. Intestinal transit time was examined in four publications, all of which concluded that such intervention was beneficial regarding bowel movement [86, 89-91].

Of note, we found some papers $(N=15)$ that covered IBS therapeutic approaches where probiotics were recommended/evaluated as a highly recommended treatment option, but the authors of the different studies examined different IBS subtypes as mixtures or did not report on improved intestinal transit time/bowel movements frequency/stool consistency [75, 87, 90, 93-104]. These documents were not placed in Tables II-IV.

Overall, there seemed to be an agreement that probiotics may improve intestinal motility, but medical authorities predominantly recommended probiotics as an integral part of treatment for constipation cautiously. The majority did not state an exact probiotic strain, optimal dose, or duration of such intervention. There was large heterogeneity among the study designs, populations, and biases present in results and therefore limited overall standard of evidence. More well-powered and high-quality trials are necessary to establish a clear consensus, with exact strains, regarding the utility of probiotic supplements in patients with constipation. It must be emphasised that a few studies did not analyse multiple variables (and therefore corrections for multiple comparisons were/are necessary) for constipation-related outcomes in IBS-C patients.

\section{Knowledge gaps}

Although the effects of probiotic therapy in constipation seem promising, there are several significant gaps in clinical knowledge:

a) Despite the aforementioned studies, strong evidence indicating a direct interaction between probiotic strains and constipation is lacking [105]. The large heterogeneity of the studies included in the systematic reviews, as well as in meta-analyses (e.g. numbers of samples, ethnicity, methodology), makes it difficult to establish a consensus or guidelines.

b) All studies evaluating intestinal microbiotic composition in constipated patients and alterations following probiotic therapy were based on faecal sample analyses. As reported by Parthasarathy et al., bacteria associated with the colonic mucosal are more predictive of constipation than the luminal populations used in most of the studies [106]. This suggests that colonic biopsy may provide more accurate material for microbiome assessment and may reveal definitive bacterial taxa related to constipation.

There is a lack of large population-based RCTs concerning adults. Current results are encouraging but limited due to the low quality of the studies.

\section{Conclusions}

Although probiotics are often recommended by medical authorities, their well-established utility in adults with constipation is uncertain. Recommendations are usually based on the results of individual studies, rather than results from meta-analyses. Additionally, meta-analyses often indicate a group of probiotics rather than individual strains, which has created difficulties for physicians making therapeutic decisions. More randomised clinical studies with FC patients, utilising well-identified strains or their combinations, are necessary to deliver a high-level of credible opinion for such intervention.

\section{Acknowledgments}

We thank Dr. Jeremy Clark for expert English proof reading in his native language.

\section{Conflict of interest}

I.Ł. and W.M. are foundation shareholders in Sanprobi, a probiotics distributor/manufacturer, and M.K. and K.S.Ż receive remuneration from this company. It should be noted that the content of this study was neither restricted nor constrained by this fact. A.K. has no conflict of interest to declare.

\section{References}

1. Lacy BE. Update on the management of chronic idiopathic constipation. Am J Manag Care 2019; 25: S55-62.

2. Wald A. Constipation: advances in diagnosis and treatment. JAMA 2016; 315: 185-91.

3. Sharma A, Rao S. Constipation: pathophysiology and current therapeutic approaches. Handb Exp Pharmacol 2017; 239: 59-74.

4. Everhart JE, Ruhl CE. Burden of digestive diseases in the United States part I: overall and upper gastrointestinal diseases. Gastroenterology 2009; 136: 376-86.

5. Peery AF, Dellon ES, Lund J, et al. Burden of gastrointestinal disease in the United States: 2012 update. Gastroenterology 2012; 143: 1179-87.e3.

6. Nellesen D, Yee K, Chawla A, et al. A systematic review of the economic and humanistic burden of illness in irritable bowel syndrome and chronic constipation. J Manag Care Pharm 2013; 19: 755-64. 
7. Nelson AD, Camilleri M, Chirapongsathorn S, et al. Comparison of efficacy of pharmacological treatments for chronic idiopathic constipation: a systematic review and network meta-analysis. Gut 2017; 66: 1611-22.

8. Black CJ, Ford AC. Chronic idiopathic constipation in adults: epidemiology, pathophysiology, diagnosis and clinical management. Med J Aust 2018; 209: 86-91.

9. Rondanelli M, Faliva MA, Perna S, et al. Using probiotics in clinical practice: where are we now? A review of existing meta-analyses. Gut Microbes 2017; 8: 521-43.

10. Marlicz W, Yung DE, Skonieczna-Żydecka K, et al. From clinical uncertainties to precision medicine: the emerging role of the gut barrier and microbiome in small bowel functional diseases. Expert Rev Gastroenterol Hepatol 2017; 11: 961-78.

11. Mogil JS, Macleod MR. No publication without confirmation. Nature 2017; 542: 409-11.

12. Hill C, Guarner F, Reid G, et al. Expert consensus document The International Scientific Association for Probiotics and Prebiotics consensus statement on the scope and appropriate use of the term probiotic. Nat Rev Gastroenterol Hepato 2014; 11: 506-14.

13. Drossman DA, Hasler WL. Rome IV - functional GI disorders: disorders of gut-brain interaction. Gastroenterology 2016 150: $1257-61$.

14. Drossman DA. Functional gastrointestinal disorders: history, pathophysiology, clinical features and Rome IV. Gastroenterology 2016. DOI: 10.1053/j.gastro.2016.02.032.

15. Mearin F, Lacy BE, Chang L, et al. Bowel disorders. Gastroenterology 2016. DOI: 10.1053/j.gastro.2016.02.031.

16. Choung RS, Locke GR, Schleck CD, et al. Cumulative incidence of chronic constipation: a population-based study 19882003. Aliment Pharmacol Ther 2007; 26: 1521-8.

17. Chang JY, Locke GR, Schleck CD, et al. Risk factors for chronic constipation and a possible role of analgesics. Neurogastroenterol Motil 2007; 19: 905-11.

18. Dukas L, Willett WC, Giovannucci EL. Association between physical activity, fiber intake, and other lifestyle variables and constipation in a study of women. Am J Gastroenterol 2003; 98: 1790-6.

19. Ge X, Zhao W, Ding C, et al. Potential role of fecal microbiota from patients with slow transit constipation in the regulation of gastrointestinal motility. Sci Rep 2017; 7: 441.

20. Brandt LJ, Prather CM, Quigley EMM, et al. Systematic review on the management of chronic constipation in North America. Am J Gastroenterol 2005; 100 Suppl 1: S5-21.

21. Ford AC, Moayyedi P, Lacy BE, et al. American College of Gastroenterology monograph on the management of irritable bowel syndrome and chronic idiopathic constipation. Am J Gastroenterol 2014; 109 Suppl 1: S2-26.

22. Lovell RM, Ford AC. Global prevalence of and risk factors for irritable bowel syndrome: a meta-analysis. Clin Gastroentero Hepatol 2012; 10: 712-21.e4.

23. Stachowska E, Maciejewska D, Ryterska K, et al. Abdominal pain and disturbed bowel movements are frequent among young people. A population based study in young participants of the Woodstock Rock Festival in Poland. J Gastrointest Liver Dis 2018; 27: 379-83.
24. Skonieczna-Zydecka K, Stachowska E, Maciejewska D, et al. The digestive health among participants of the Woodstock Rock Festival in Poland - a cross-sectional survey. Int J Environ Res Public Health 2018; 15. DOI: 10.3390/ ijerph15102256.

25. Shekhar C, Monaghan PJ, Morris J, et al. Rome III functional constipation and irritable bowel syndrome with constipation are similar disorders within a spectrum of sensitization, regulated by serotonin. Gastroenterology 2013; 145: 749-57.

26. Wong RK, Palsson OS, Turner MJ, et al. Inability of the Rome III criteria to distinguish functional constipation from constipation-subtype irritable bowel syndrome. Am J Gastroenterol 2010; 105: 2228-34

27. Zhao YF, Ma XQ, Wang R, et al. Epidemiology of functional constipation and comparison with constipation-predominant irritable bowel syndrome: the Systematic Investigation of Gastrointestinal Diseases in China (SILC). Aliment Pharmacol Ther 2011; 34: 1020-9.

28. Quigley EMM. Editorial: differentiating chronic idiopathic constipation from constipation-predominant irritable bowel syndrome: possible and important? Aliment Pharmacol Ther 2015; 41: 1299.

29. Tack J, Drossman DA. What's new in Rome IV? Neurogastroenterol Motil 2017; 29. DOI: 10.1111/nmo.13053.

30. Simren M, Palsson OS, Whitehead WE. Update on Rome IV criteria for colorectal disorders: implications for clinical practice. Curr Gastroenterol Rep 2017; 19: 15.

31. Chan AOO, Lam KF, Hui WM, et al. Influence of positive family history on clinical characteristics of functional constipation. Clin Gastroenterol Hepatol 2007; 5: 197-200.

32. Ostwani W, Dolan J, Elitsur Y. Familial clustering of habitual constipation: a prospective study in children from West Virginia. J Pediatr Gastroenterol Nutr 2010; 50: 287-9.

33. Camilleri M, Vazquez-Roque MI, Carlson P, et al. Association of bile acid receptor TGR5 variation and transit in health and lower functional gastrointestinal disorders. Neurogastroenterol Motil 2011; 23: 995-9, e458.

34. Beyder A, Mazzone A, Strege PR, et al. Loss-of-function of the voltage-gated sodium channel NaV1.5 (channelopathies) in patients with irritable bowel syndrome. Gastroenterology 2014; 146: 1659-68.

35. Tzavella K, Riepl RL, Klauser AG, et al. Decreased substance $P$ levels in rectal biopsies from patients with slow transit constipation. Eur J Gastroenterol Hepatol 1996; 8: 1207-11.

36. Cortesini C, Cianchi F, Infantino A, et al. Nitric oxide synthase and VIP distribution in enteric nervous system in idiopathic chronic constipation. Dig Dis Sci 1995; 40: 2450-5.

37. El-Salhy M, Norrgård O, Spinnell S. Abnormal colonic endocrine cells in patients with chronic idiopathic slow-transit constipation. Scand J Gastroenterol 1999; 34: 1007-11.

38. He CL, Burgart L, Wang L, et al. Decreased interstitial cell of cajal volume in patients with slow-transit constipation. Gastroenterology 2000; 118: 14-21.

39. Lyford GL, He CL, Soffer E, et al. Pan-colonic decrease in interstitial cells of Cajal in patients with slow transit constipation. Gut 2002; 51: 496-501.

40. Lynch SV, Pedersen O. The human intestinal microbiome in health and disease. N Engl J Med 2016; 375: 2369-79. 
41. Felice VD, O'Mahony SM. The microbiome and disorders of the central nervous system. Pharmacol Biochem Behav 2017; 160: 1-13.

42. Sender R, Fuchs S, Milo R. Revised estimates for the number of human and bacteria cells in the body. PLoS Biol 2016; 14 e1002533.

43. Gallagher J. More than half your body is not human. BBC News 2018, https://www.bbc.com/news/health-43674270.

44. Ohkusa T, Koido S, Nishikawa Y, et al. Gut microbiota and chronic constipation: a review and update. Front Med (Lausanne) 2019; 6: 19.

45. Linetzky Waitzberg D, Alves Pereira CC, Logullo L, et al. Microbiota benefits after inulin and partially hydrolized guar gum supplementation: a randomized clinical trial in constipated women. Nutr Hosp 2012; 27: 123-9.

46. Khalif IL, Quigley EMM, Konovitch EA, et al. Alterations in the colonic flora and intestinal permeability and evidence of immune activation in chronic constipation. Dig Liver Dis 2005; 37: 838-49.

47. Kim SE, Choi SC, Park KS, et al. Change of fecal flora and effectiveness of the short-term VSL\#3 probiotic treatment in patients with functional constipation. J Neurogastroenterol Motil 2015; 21: 111-20.

48. Liśkiewicz P, Pełka-Wysiecka J, Kaczmarczyk M, et al. Fecal microbiota analysis in patients going through a depressive episode during treatment in a psychiatric hospital setting. J Clin Med 2019; 8: piiE164.

49. Malla MA, Dubey A, Kumar A, et al. Exploring the human microbiome: the potential future role of next-generation sequencing in disease diagnosis and treatment. Front Immunol 2019; 9: 2868.

50. Langille MGI, Zaneveld J, Caporaso JG, et al. Predictive functional profiling of microbial communities using 16S rRNA marker gene sequences. Nature Biotechnology 2013; 31: 814-21.

51. Skonieczna-Żydecka K, Grochans E, Maciejewska D, et al. Faecal short chain fatty acids profile is changed in polish depressive women. Nutrients 2018; 10: pii: E1939.

52. Kennedy PJ, Clarke G, Quigley EMM, et al. Gut memories: towards a cognitive neurobiology of irritable bowel syndrome. Neurosci Biobehav Rev 2012; 36: 310-40.

53. Rhee SH, Pothoulakis C, Mayer EA. Principles and clinical implications of the brain-gut-enteric microbiota axis. Nat Rev Gastroenterol Hepatol 2009; 6: 306-14.

54. Boeckxstaens G, Camilleri M, Sifrim D, et al. Fundamentals of neurogastroenterology: physiology/motility - sensation. Gastroenterology 2016. DOI: 10.1053/j.gastro.2016.02.030.

55. Obata Y, Pachnis V. The effect of microbiota and the immune system on the development and organization of the enteric nervous system. Gastroenterology 2016; 151: 836-44.

56. Barbara G, Stanghellini V, Brandi G, et al. Interactions between commensal bacteria and gut sensorimotor function in health and disease. Am J Gastroenterol 2005; 100: 2560-8.

57. Dembinski A, Warzecha Z, Konturek PJ, et al. Influence of capsaicin-sensitive afferent neurons and nitric oxide (NO) on cerulein-induced pancreatitis in rats. Int J Pancreatol 1996; 19: 179-89.

58. Dembiński A, Warzecha Z, Ceranowicz P, et al. The role of capsaicin-sensitive sensory neurons and nitric oxide in regu- lation of gastric mucosal growth. J Physiol Pharmacol 1995; 46: 351-62.

59. Holzer P, Farzi A. Neuropeptides and the microbiota-gut-brain axis. Adv Exp Med Biol 2014; 817: 195-219.

60. Pimentel M, Lin HC, Enayati P, et al. Methane, a gas produced by enteric bacteria, slows intestinal transit and augments small intestinal contractile activity. Am J Physiol Gastrointest Liver Physiol 2006; 290: G1089-95.

61. Carbonero F, Benefiel AC, Gaskins HR. Contributions of the microbial hydrogen economy to colonic homeostasis. Nat Rev Gastroenterol Hepatol 2012; 9: 504-18.

62. Teague B, Asiedu S, Moore PK. The smooth muscle relaxant effect of hydrogen sulphide in vitro: evidence for a physiological role to control intestinal contractility. Br J Pharmacol 2002; 137: 139-45.

63. McCusker RH, Kelley KW. Immune-neural connections: how the immune system's response to infectious agents influences behavior. J Exp Biol 2013; 216: 84-98.

64. Hoyles L, Snelling T, Umlai UK, et al. Microbiome-host systems interactions: protective effects of propionate upon the bloodbrain barrier. Microbiome 2018; 6: 55.

65. Zhao Y, Yu YB. Intestinal microbiota and chronic constipation. Springerplus 2016; 5: 1130.

66. Floch $\mathrm{MH}$. Bile salts, intestinal microflora and enterohepatic circulation. Dig Liver Dis 2002; 34 Suppl 2: S54-57.

67. Kang DW, DiBaise JK, Ilhan ZE, et al. Gut microbial and shortchain fatty acid profiles in adults with chronic constipation before and after treatment with lubiprostone. Anaerobe 2015; 33: 33-41.

68. Abrahamsson H, Ostlund-Lindqvist A-M, Nilsson R, et al. Altered bile acid metabolism in patients with constipation-predominant irritable bowel syndrome and functional constipation. Scand J Gastroenterol 2008; 43: 1483-8.

69. Mayer EA. The neurobiology of stress and gastrointestinal disease. Gut 2000; 47: 861-9.

70. Quigley EMM. The spectrum of small intestinal bacterial overgrowth (SIBO). Curr Gastroenterol Rep 2019; 21: 3.

71. Roland BC, Ciarleglio MM, Clarke JO, et al. Small intestinal transit time is delayed in small intestinal bacterial overgrowth. J Clin Gastroenterol 2015; 49: 571-6.

72. Sarosiek I, Bashashati M, Alvarez A, et al. Lubiprostone accelerates intestinal transit and alleviates small intestinal bacterial overgrowth in patients with chronic constipation. Am J Med Sci 2016; 352: 231-8.

73. Grover M, Kanazawa M, Palsson OS, et al. Small intestinal bacterial overgrowth in irritable bowel syndrome: association with colon motility, bowel symptoms, and psychological distress. Neurogastroenterol Motil 2008; 20: 998-1008.

74. Agrawal A, Houghton LA, Morris J, et al. Clinical trial: the effects of a fermented milk product containing Bifidobacterium lactis DN-173 010 on abdominal distension and gastrointestinal transit in irritable bowel syndrome with constipation. Aliment Pharmacol Ther 2009; 29: 104-14.

75. Pietrzak A, Skrzydło-Radomańska B, Mulak A, et al. Guidelines on the management of irritable bowel syndrome. Gastroenterology Rev 2018; 13: 259-88.

76. Valdovinos MA, Montijo E, Abreu AT, et al. The Mexican consensus on probiotics in gastroenterology. Rev Gastroenterol Méx 2017; 82: 156-78. 
77. Skokovic-Sunjic D. Clinical Guide to probiotic products available in Canada. www.probioticchart.ca 2018.

78. Clinical Guide to Probiotic Products Available in Canada. 2019 Edition indications for adult health.

79. Clinical Guide to Probiotic Products Available in the United States: 2019 Edition Indications for Adult Health.

80. Guarner F, Sanders ME, Eliakim R, et al. Probiotics and prebiotics. World Gastroenterology Organisation Global Guidelines, http://www.worldgastroenterology.org/UserFiles/file/ guidelines/probiotics-and-prebiotics-english-2017.pdf (2017)

81. Hungin APS, Mulligan C, Pot B, et al. Systematic review: probiotics in the management of lower gastrointestinal symptoms in clinical practice - an evidence-based international guide. Alimentary Pharmacol Ther 2013; 38: 864-86.

82. Hungin APS, Mitchell CR, Whorwell P, et al. Systematic review: probiotics in the management of lower gastrointestinal symptoms - an updated evidence-based international consensus. Alimentary Pharmacol Ther 2018; 47: 1054-70.

83. Andresen V, Keller J, Pehl C, et al. Irritable bowel syndrome. Dtsch Arztebl Int 2011; 108:751-60. .

84. Mearin F, Ciriza C, Mínguez M, et al. Clinical Practice Guideline: irritable bowel syndrome with constipation and functional constipation in the adult. Rev Esp Enferm Dig 2016; 108: 332-63.

85. Chmielewska A, Szajewska H. Systematic review of randomised controlled trials: probiotics for functional constipation. World J Gastroenterol 2010; 16: 69-75.

86. Dimidi E, Christodoulides S, Fragkos KC, et al. The effect of probiotics on functional constipation in adults: a systematic review and meta-analysis of randomized controlled trials. Am J Clin Nutr 2014; 100: 1075-84.

87. Ford AC, Harris LA, Lacy BE, et al. Systematic review with metaanalysis: the efficacy of prebiotics, probiotics, synbiotics and antibiotics in irritable bowel syndrome. Aliment Pharmacol Ther 2018; 48: 1044-60.

88. Martínez-Martínez MI, Calabuig-Tolsá R, Cauli O. The effect of probiotics as a treatment for constipation in elderly people: a systematic review. Arch Gerontol Geriatr 2017; 71: 142-9.

89. Miller LE. Effects of probiotic-containing products on stool frequency and intestinal transit in constipated adults: systematic review and meta-analysis of randomized controlled trials. Ann Gastroenterol 2017; 30: 629-39.

90. McKenzie YA, Thompson J, Gulia P, et al. British Dietetic Association systematic review of systematic reviews and evidence-based practice guidelines for the use of probiotics in the management of irritable bowel syndrome in adults (2016 update). J Hum Nutr Diet 2016; 29: 576-92.

91. Miller LE. Probiotic supplementation decreases intestina transit time: meta-analysis of randomized controlled trials. World J Gastroenterol 2013; 19: 4718-25.

92. Ortiz-Lucas M, Tobías A, Saz P, et al. Effect of probiotic species on irritable bowel syndrome symptoms: a bring up to date meta-analysis. Rev Esp Enferm Dig 2013; 105: 19-36.

93. Brenner DM, Moeller MJ, Chey WD, et al. The utility of probiotics in the treatment of irritable bowel syndrome: a systematic review. Am J Gastroenterol 2009; 104: 1033-49.

94. Corbitt M, Campagnolo N, Staines D, et al. A systematic review of probiotic interventions for gastrointestinal symptoms and irritable bowel syndrome in chronic fatigue syndrome/ myalgic encephalomyelitis (CFS/ME). Probiotics Antimicrob Proteins 2018; 10: 466-77.

95. Didari T. Effectiveness of probiotics in irritable bowel syndrome: updated systematic review with meta-analysis. World J Gastroenterol 2015; 21: 3072-84.

96. Floch $\mathrm{MH}$, Walker WA, Madsen K, et al. Recommendations for probiotic use-2011 update. J Clin Gastroenterol 2011; 45 Suppl: S168-71.

97. Floch M. Recommendations for probiotic use in humans a 2014 update. Pharmaceuticals 2014; 7: 999-1007.

98. Ford AC, Moayyedi P, Chey WD, et al. American College of Gastroenterology Monograph on management of irritable bowel syndrome. Am J Gastroenterol 2018; 113 (suppl 2): 1-18.

99. Dimidi E, Cox C, Scott SM, Whelan K. Probiotic use is common in constipation, but only a minority of general and specialist doctors recommend them and consider there to be an evidence base. Nutrition 2016; 61: 157-63.

100. Guarner F, Khan AG, Garisch J; World Gastroenterology Organization. World Gastroenterology Organisation Global Guidelines: probiotics and prebiotics October 2011. J Clin Gastroenterol 2012; 46: 468-81.

101. Hoveyda N, Heneghan C, Mahtani KR, et al. A systematic review and meta-analysis: probiotics in the treatment of irritable bowel syndrome. BMC Gastroenterology 2009; 9: 15.

102. Song KH, Jung HK, Kim HJ, et al. Clinical practice guidelines for irritable bowel syndrome in Korea, 2017 revised edition. J Neurogastroenterol Motil 2018; 24: 197-215.

103. World Gastroenterology Organisation practice guideline: probiotics and prebiotics. Arab J Gastroenterol 2009; 10: 33-42.

104. Zhang Y, Li L, Guo C, et al. Effects of probiotic type, dose and treatment duration on irritable bowel syndrome diagnosed by Rome III criteria: a meta-analysis. BMC Gastroenterology 2016; 16: 62.

105. Choi $\mathrm{CH}$, Chang SK. Alteration of gut microbiota and efficacy of probiotics in functional constipation. J Neurogastroenterol Motil 2015; 21: 4-7.

106. Parthasarathy G, Chen J, Chen X, et al. Relationship between microbiota of the colonic mucosa vs feces and symptoms, colonic transit, and methane production in female patients with chronic constipation. Gastroenterology 2016; 150: 367-79.e1.

Received: 28.04.2019

Accepted: 3.06.2019 